\title{
The five year outcome of severe blunt head injury: a relative's view
}

\author{
N BROOKS, L CAMPSIE, C SYMINGTON, A BEATTIE, W MCKINLAY \\ From the University Department of Psychological Medicine, Glasgow, UK
}

SUMMARY A close relative of each of 42 severely head injured patients was interviewed at 5 years after injury, following initial study at 3, 6, and 12 months. Persisting severe deficits, in some cases worse than at 1 year, were primarily psychological and behavioural, although minor physical deficits, for example in vision, were also common. Relatives were under great strain; significantly more so than at 1 year. The best predictor of strain in the relative was the magnitude of behavioural and personality change in the patient.

The purely physical sequelae of severe head injury (sensorimotor disturbance, gait disturbance, cranial nerve lesions, etc) are well documented, ${ }^{12}$ but it is becoming increasingly recognised that often the most serious long term morbidity after head injury is psychological; involving cognitive, behavioural, and social and family disturbance.

Research into psychological sequelae has produced reports of both short term (1-2 years), ${ }^{3-9}$ and long term $^{10-17}$ outcome. The reports are based on widely varying samples of patients ranging from those admitted to one neurosurgical unit, ${ }^{3}$ to those admitted to a variety of hospital units, ${ }^{7}$ and those in a rehabilitation unit. ${ }^{81314}$ Despite this variation, and with rare exceptions, for example ref 16 , the consensus is that in the more severely injured patient, psychological morbidity in terms both of serious behavioural change in the patient, and distress in the family is initially very high. ${ }^{341819}$ Furthermore, the morbidity may persist long after injury. ${ }^{12-1417}$ Nevertheless, few studies have carried out a late analysis of cases examined in detail early after injury. (For exceptions see refs 13 , and 17). This dearth has meant that the early identification of predictors of late outcome is still problematical, and it is difficult to specify the mechanisms of late morbidity (for example whether related solely to severity of brain damage, or to premorbid, or other non organic factors).

Israeli workers ${ }^{13}$ serially assessed patients in an intensive rehabilitation programme early after injury, showing that 1-6 years after injury, the patients who were still most severely behaviourally impaired were

Address for reprint requests: Professor N Brooks, 6 Whittinghame Gardens, Gt Western Road, Glasgow G120AA, UK.

Received 27 September 1985 and in revised form 20 November 1985. Accepted 23 November 1985 those with the most severe brain damage. ${ }^{17}$ Thomsen's 10-15 year follow-up of cases initially examined within 2 years of injury, disclosed a high incidence of divorce, continuing dependency, communication disturbance, and psychosocial sequelae including personality change and emotional disturbance. These late sequelae related to the presence and degree of brain stem damage (presumably reflecting the severity of damage throughout the cerebrum).

The present authors reported a detailed study of psychosocial outcome 1 year after injury, ${ }^{3}$ in which attempts were made to describe the natural history of psychosocial disturbance in patient and family, and to relate changes in the patient to distress in the family. By one year after injury emotional and behavioural disturbances in the patient were frequently described by a relative, and these rather than continuing physical or communication disturbances were the best predictors of stress in the relative who had the main responsibility for caring for the patient. The present study reports the 5 year outcome of a sample of these patients. The aims were firstly to identify the patterns of change in behavioural disturbance in the patient and distress in the family during the first 5 years after injury; and secondly, to identify predictors of behavioural disturbance in the patient and continuing distress in the caring relative.

\section{Method}

\section{Patients}

The patient population from which the current study sample was drawn comprised 55 cases ( 46 male), aged 16-60 years at time of injury, with a mean age of 35.7 years (SD 14.3). All had suffered a severe blunt head injury defined by at least 2 days post traumatic amnesia (PTA, assessed retrospectively by careful clinical questioning). Cases with purely pene- 
Table 1 Details of patients and relatives

\begin{tabular}{|c|c|c|c|c|c|}
\hline \multicolumn{4}{|l|}{ Patients } & \multicolumn{2}{|l|}{ Relatives } \\
\hline Age (yr) & $N$ & $P T A^{*}$ & $N$ & Relationship & $N$ \\
\hline $\begin{array}{l}\text { Under } 25 \\
26-30 \\
31-40 \\
41-50 \\
51-64\end{array}$ & $\begin{array}{r}9 \\
8 \\
7 \\
7 \\
11 \\
42\end{array}$ & $\begin{array}{l}2-14 \text { days } \\
15-28 \text { days } \\
1-2 \text { months } \\
\text { Over } 2 \text { months } \\
\text { No reliable data }\end{array}$ & $\begin{array}{r}16 \\
9 \\
8 \\
7 \\
2 \\
42\end{array}$ & $\begin{array}{l}\text { Wife } \\
\text { Husband } \\
\text { Mother } \\
\text { Father } \\
\text { Other }\end{array}$ & $\begin{array}{r}19 \\
4 \\
7 \\
5 \\
7 \\
42\end{array}$ \\
\hline
\end{tabular}

*PTA $=$ Post traumatic amnesia

trating focal wounds were excluded, although those with depressed fractures were not excluded. Patients had all been referred to the Institute of Neurological Sciences, Glasgow, for neurosurgical management, and comprised therefore a severe sample with many patients having intracranial operations such as the evacuation of haematoma. More detailed information is available in the original publication describing these cases. ${ }^{3}$

Five years after injury an attempt was made to contact all 55 cases, and a success rate of $76 \%$ was achieved, giving 42 patients (36 male). The 13 cases not included comprised three who could not be located, five who had died, four who were unwilling to take part in further research, and one who was found to have been suffering from epilepsy for many years prior to his injury. (For this reason he should not have been included in the first study: however, after his exclusion reanalysis of all the one year results reported in the previous study ${ }^{3}$ gave virtually identical results).

The mean age of the sample (table 1) at the 5 year followup was 36 years (22-64 years). The 42 cases appear to be highly representative of the original 55 , containing similar distributions of type of accident, similar PTA distributions, and similar distributions of cases with fracture and/or haematoma. As before, the lower social classes were over represented with 21 cases belonging to social class 4 or 5; and the sample is a very severely injured one (table 1) with 24 of the 42 cases $(60 \%)$ having a PTA of 15 days or more $(66 \%$ previously), and a median PTA of 21 days, identical to that in the previous study.

\section{Procedure}

A procedure identical to that in the one year study was used. A close relative was interviewed (table 1) and asked to report any changes in the patient which had emerged after injury, and which were still present. An attempt was made to interview the same relative who had been seen at one year, but this was not always possible, and in eight cases a different (although still a close) relative was interviewed. A structured interview schedule was used to assess both patient and family member, although this paper concerns only the report of the family member.

The interview included questions about the patient's physical and mental state, behaviour, self care abilities, and personality. As in the one year study, an attempt was made to relate changes in the patient to the degree of burden perceived by the relative, and a model of burden was used in which change in the patient was designated "objective" burden, and the amount of strain or distress experienced by the relative as arising from these objective changes was desig- nated "subjective" burden. ${ }^{20}$ Subjective burden was assessed by a variety of different methods (for example self report of distress, visual analogue scale, relative's reports of her/his own medical consultations and medications, etc), but for the purposes of this study only one method is reported: a 7 point rating scale ranging from the low point "I feel no strain as a result of changes in my spouse/relative", to the maximum of "I feel severe strain...".

\section{Results}

The data were analysed firstly to assess the frequency of continuing problems in the patient as reported by the relative, and secondly to identify predictors of continuing disturbance in the patient. The magnitude of subjective burden in the relative was then assessed, and finally an attempt was made to identify changes in the patient which best predicted the magnitude of burden in the relative. For a discussion of the pros and cons of taking a relative's report rather than that of the patient, see ref 21 .

\section{(1)}

\section{CONTINUING PROBLEMS IN THE PATIENT}

Relatives were asked to report the presence or absence of a variety of behavioural, affective, psychological, and physical changes in the patient. The ten most frequently reported are shown in table 2 , which also indicates the item frequency at one year. In interpreting the results it must be borne in mind that the

Table 2 The ten problems most frequently reported by relatives at 5 years $(N=42)$

\begin{tabular}{lll}
\hline Problem & \multicolumn{2}{l}{ Per cent relatives reporting } \\
\cline { 2 - 3 } & 1 year & 5 years \\
\hline Personality change & 60 & 74 \\
Slowness & 65 & 67 \\
Poor memory & 67 & 67 \\
Irritability & 67 & 64 \\
Bad temper & 64 & 64 \\
Tiredness & 69 & 62 \\
Depression & 51 & 57 \\
Rapid mood change & 57 & 57 \\
Tension and anxiety & 57 & 57 \\
Threats of violence & 15 & 54 \\
\hline
\end{tabular}


Table 3 Mean number of difficulties (out of 10) reported by relatives at 5 years

\begin{tabular}{lll}
\hline Difficulty & 1 year & 5 years \\
\hline Physical & $2 \cdot 3$ & $2 \cdot 6$ \\
Language & $2 \cdot 0$ & $2 \cdot 8$ \\
Emotion & $5 \cdot 0$ & $5 \cdot 2$ \\
Dependence & $1 \cdot 3$ & $2 \cdot 3$ \\
Subjective & $4 \cdot 3$ & $4 \cdot 5$ \\
Memory & $1 \cdot 6$ & $2 \cdot 9$ \\
Disturbed behaviour & $2 \cdot 2$ & $3 \cdot 7$ \\
\hline
\end{tabular}

percentages at the two times refer to the population sample of 42 cases, and for this reason the one year figures are not identical to those reported in the previous publication, ${ }^{3}$ which refer to the original 55 cases.

At 5 years just as at one year, the most frequently reported items were in the broadly "mental", or "behavioural" area. The single most frequent report at 5 years was that of continuing personality change which had increased from $60 \%$ to $74 \%$. The next most frequent reports involved slowness, memory, irritability, and bad temper, all of which had been reported by at least $64 \%$ of relatives at one year. Most of the "top ten" high frequency 5 year items had changed by no more than a few percentage points from the one year figure. However, two problems were reported to increase markedly (personality change and threats of violence), with the latter item showing a dramatic increase from $15 \%$ to $54 \%$. Two of the problems had reduced in frequency, although by only 3 and 7 percentage points respectively (irritability and tiredness).

Having identified specific high frequency items, an overview of the most prevalent difficulties at 5 years was obtained by subdividing questionnaire items into the 7 broad categories reported in the previous study. For each category the results were scaled to give a maximum of 10 , and the results (table 3 ) are as follows:

(1) Physical The mean number of physical difficulties was similar to that at one year. Gross physical disturbances were unusual, even one year after injury, when $88 \%$ of patients were fully independently mobile ( $93 \%$ at 5 years). Nevertheless, subtle difficulties involving minor problems with vision (unspecified) were still present ( $41 \%$ compared with $43 \%$ at one year) as were continuing problems of balance ( $48 \%$ compared with $40 \%$ at one year).

(2) Language The overall level of language disturbance had increased from a mean of 2.0 to 2.8 , largely owing to an increased reporting of dysarthria $(41 \% ; 29 \%$ at one year). Thirty three per cent of patients were still reported to have difficulties in linguistic expression ( $44 \%$ at one year), but the problems both in expression and dysarthria had little functional impact upon the patient, and many relatives pointed out that problems were not continually present, being obvious only when the patient was tired or upset.

(3) Emotion Emotional changes were reported very frequently both at one year $(5 \cdot 0)$ and $(5 \cdot 2)$ at 5 years. All individual items which had been reported at one year (irritability, tension/anxiety, temper, etc) were still reported to be present in over half the patients, and some items (for example personality change), were reported to have increased very considerably.

(4) Dependence This category included self care; the need for the patient to be supervised; and the patient's ability to undertake responsibility, for example undertaking care of the children or taking charge of the household. The overall level of disturbance was reported to have increased from a mean of $1 \cdot 3$ to $2 \cdot 3$. Self care difficulties were not frequent: only six patients $(14 \%)$ needed any help with washing and dressing, and these were patients with very prolonged durations of PTA. However, $21 \%$ of relatives reported that the patient needed someone to look after him at home, and nearly a half $(43 \%$, contrasting with the $18 \%$ at one year) reported that the patient could not be left in charge of the household. It was very rare that major accidents or disasters had happended when the patient was left in charge of the household, but many relatives felt that the patient lacked the judgement to respond rapidly and effectively to an emergency.

(5) Subjective The 5 year picture was similar to that at one year. Over half the relatives reported slowness $(67 \%)$, tiredness $(62 \%)$, and concentration problems $(52 \%)$. The figures for one year were $64 \%, 69 \%$, and $36 \%$ respectively. Although the incidence of concentration problems has increased, other problems (headaches and inability to tolerate noise) dropped (55\% to $41 \%$; and $46 \%$ to $36 \%$ respectively).

(6) Memory The overall level of continuing memory problems had increased from a mean of 1.6 to 2.9 . Particular changes were found in reports of the patient forgetting what he was doing in the middle of an action sequence $(47 \%$, up from $17 \%)$; repeating or double checking actions (45\% from $35 \%$ ); and losing track of what he was saying ( $42 \%$ from $33 \%)$. However, many relatives felt that the memory problem did not have a serious impact upon the patient's day to day life.

(7) Disturbed behaviour This category representing a broad range of difficult behaviours, including bizarre, puzzling, violent, or inappropriate social behaviour; had increased greatly from a mean of 2.0 to 3.7 . At 5 years the most frequently reported problem was threats or gestures of violence $(54 \% ; 15 \%$ at one year), and 1 in $5(20 \%)$ relatives reported that the patient had been physically violent involving an ac- 
Table 4 Product moment correlations between patient difficulties and PTA at 1 and 5 years (Numbers of cases in parentheses)

\begin{tabular}{lll}
\hline Difficulty & 1 year & 5 years \\
\hline Physical & $0.38(40)^{*}$ & $0.28(40)$ \\
Language & $0.38(41)$ & $0.31(40)$ \\
Emotion & $0.22(39)$ & $0.22(40)$ \\
Dependence & $0.51(40)^{*}$ & $0.53(40)^{*}$ \\
Subjective & $0.15(39)$ & $0.26(40)$ \\
Memory & $0.47(36)^{*}$ & $0.26(36)$ \\
Disturbed behaviour & $0.38(34)^{*}$ & $0.32(23)$ \\
\hline
\end{tabular}

${ }^{*} \mathrm{p}<0.01$.

tual assault on the relative ( $10 \%$ at one year). In addition, 13 patients $(31 \% ; 7 \%$ at one year) had been in some trouble with the law since their accident; the offences ranging from breach of the peace through drunk and disorderly charges, motoring offences, and one attempted murder charge. Eight of this group had had at least some brush with the law prior to injury, and the appearance of these late offences is therefore difficult to attribute wholly or even in part to the injury. Further continuing problems included childishness (38\%; $46 \%$ at 1 year); talking too much (38\%; $27 \%$ at 1 year); and being readily upset even by small changes in routine $(38 \% ; 24 \%$ at 1 year). The problems in this broad category have nearly all increased.

\section{PREDICTION OF PERSISTING PROBLEMS IN THE PATIENT}

In view of the varying pattern of change in problems over time (some increasing, some diminishing, and some not changing), an attempt was made to predict continuing disturbance at 5 years. An obvious predictor is the severity of brain damage, although this is unlikely to bear the same degree of relationship to all aspects of outcome, in view of the differing natural histories of the different outcome categories.

The association between continuing problems and severity of brain damage was examined by means of Pearson Product Moment correlations computed between the duration of post traumatic amnesia and the levels of deficit in each of the seven problem areas. A significance level of 0.01 rather than the conventional 0.05 was taken, as 14 separate coefficients were computed. As the seven areas of continuing disturbance comprise composite scales, missing data points are a problem. If any individual item from the category was missing, the patient in question was excluded entirely from the analysis giving the numbers indicated in parenthesis in table 4.

At 1 year, 4 , and at 5 years, 1 of the 7 coefficients reached statistical significance. Different aspects of outcome in the patient as judged by a relative do not therefore relate equally to severity of injury. Furthermore, as time progresses, the extent to which severity predicts outcome reduces, so that by 5 years only the more subtle dependency aspects of outcome related significantly to severity of brain damage. Neither affective and emotional aspects of outcome, nor physical, behavioural, or cognitive aspects of outcome relate to severity at 5 years.

(3)

\section{BURDEN UPON THE RELATIVES}

The level of subjective burden (SB) experienced by relatives was assessed on the seven point scale described in the Procedure section. In three cases SB data were unavailable. The seven point scale was categorised into three levels of burden as follows: Low burden (SB ratings 1-2); Medium burden (SB ratings 3-4); High burden (SB ratings 5-7). These correspond exactly to the "stress" ratings reported in the one year follow-up.

As table 5 shows, the distribution of burden had changed from 1-5 years with a preponderance of Low to Medium burden at one year, and a preponderance of Medium to High at 5 years. Comparison of the 39 cases with SB data at both times showed that only two of the 10 relatives with High burden at 1 year showed a reduction; whereas 18 of the 29 with Low or Medium burden at 1 year, increased to High burden at 5 years. A McNemar test for the significance of change of two categories (High compared with Low and Medium) over the two time intervals gave a Chi square of 11.5 (df l) significant at $p<0.01$ showing that the distribution of burden had indeed shifted significantly towards high levels at 5 years.

\section{(4)}

PREDICTION OF BURDEN UPON THE RELATIVE Having identified the overall magnitude of burden, an attempt was made to predict the relative's burden at 5 years firstly in terms of the severity of patient injury, and secondly, in terms of the type and magnitude of persisting problems in the patient. Injury severity was defined in terms of PTA, and one way analyses of variance were used to compare the mean durations of post traumatic amnesia in the three subjective burden

Table 5 Number of relatives in each subjective burden category at 1 and 5 years

\begin{tabular}{|c|c|c|c|c|c|}
\hline \multicolumn{2}{|c|}{ Subjective burden } & \multicolumn{2}{|c|}{1 year } & \multicolumn{2}{|c|}{5 years } \\
\hline Score & Description & $N$ & $\%$ & $N$ & $\%$ \\
\hline $\begin{array}{l}1-2 \\
3-4 \\
5-7\end{array}$ & $\begin{array}{l}\text { "Low" } \\
\text { "Medium" } \\
\text { "High" }\end{array}$ & $\begin{array}{l}18 \\
14 \\
10 \\
42\end{array}$ & $\begin{array}{l}43 \\
33 \\
24\end{array}$ & $\begin{array}{r}4 \\
13 \\
22 \\
39\end{array}$ & $\begin{array}{l}10 \\
33 \\
56\end{array}$ \\
\hline
\end{tabular}


Table 6 One way analysis of variance to compare mean PTA durations in patients as a function of subjective burden in the relative

\begin{tabular}{llcll}
\hline $\begin{array}{l}\text { Subjective } \\
\text { burden group }\end{array}$ & \multicolumn{4}{l}{ Mean PTA (days) } \\
\cline { 2 - 5 } & 1 year & $N$ & 5 years & $N$ \\
\hline "Low" & 19.6 & 18 & 12.0 & 4 \\
"Medium" & 33.3 & 14 & 18.6 & 12 \\
"High" & 44.7 & 10 & 41.3 & 21 \\
F (df) & $2.94(2.37)$ & & $3.99(2.34)$ & \\
p & 0.06 & & 0.03 & \\
\hline
\end{tabular}

groups just described. Table 6 shows the results together with those at one year. Although appropriate statistically, the analysis of variance was rather marginal at 5 years owing to the very few (4) cases in the Low burden category. Nevertheless, at that time the mean levels of PTA showed a striking difference between the three burden groups, with patients whose relatives were under Low burden having a mean PTA of 12 days, and those whose relatives had a High degree of burden having a mean PTA of 41 days $(p=$ 0.03 ). The $F$ ratio at 1 year just failed to reach significance although the mean PTA increased markedly with increasing SB.

Simple severity of injury was, therefore, one predictor of burden in the relative at 5 years, but this must, of course, be via some intermediate mechanism involving specific changes in the patient. This was investigated using the seven categories of behaviour change in the patient already described, and using one way analysis of variance or $t$ test to assess the mean magnitude of each category of behaviour in the patient, as a function of the three levels (two in the case of $t$ test) of subjective burden in the relative (table 7).

Relatives were subdivided into the three burden categories already described, although just as for the PTA analysis, numbers in the Low burden group were very small. Nevertheless, on only two of the items at 5 years (physical and memory) was there no significant relationship between the magnitude of change in the patient and the degree of burden in the relative (table 7). Just as at one year, therefore, the greater the magnitude of behavioural/ personality/affective change in the patient the greater the burden on the relative.

In view of the very small numbers in the Low burden group, $(N=4)$, the data were re-analysed using the $t$ test to compare the magnitude of behaviour change in the patient in the High burden relatives with that in the Low and Medium relatives combined. The results were identical to those gained using the analysis of variance.

\section{Discussion}

This study attempted to chart the natural history (over a 5 year period) of the objective and subjective burden consequences of severe head injury, and to identify simple predictors of both types of burden. At one year, relatives reported high levels of behavioural, personality, and cognitive change in the injured patient, although physical changes were much less prominent. Relatives suffered distress (subjective burden) attributable to the patient's injury, and the greater the personality and behavioural change in the patient, the greater the distress in the relative.

In many respects, the picture at 5 years is very similar to (or worse than) that at 1 year. The "top ten" items of change in the patient at 5 years are similar to those at 1 year, and are all broadly psychological rather than physical, reflecting increasing reports of personality change, tension, tiredness, mood change, and severe difficulties in the control of irritability and temper. A very similar picture emerged when broad categories of patient outcome rather than single symptomatic items were studied. Both on single items, and composite outcome scales, the 5 year picture showed little improvement in patient outcome, and in some cases, a marked deterioration.

For the relative, the situation deteriorated markedly. This was indicated by the increase in sub-

Table 7 Summary of one way analyses of variance ( $p$ values) of mean level of difficulty in patients as a function of subjective burden in the relative

\begin{tabular}{lllll}
\hline Difficulty & Probability level & & & \\
\cline { 2 - 5 } & 1 year & $N$ & 5 years & $N$ \\
\hline Physical & $\mathrm{p}=0.02$ & 41 & $\mathrm{NS}$ & 39 \\
Language & $\mathrm{p}=0.02$ & 42 & $\mathrm{p}=0.04$ & 39 \\
Emotion & $\mathrm{p}<0.01$ & 40 & $\mathrm{p}=0.02$ & 39 \\
Dependence & $\mathrm{NS}<0.01$ & 41 & $\mathrm{p}=0.02$ & 39 \\
Subjective & $\mathrm{p} S$ & $\mathrm{p}=0.05$ & 35 \\
Memory & $\mathrm{p}<0.01$ & 36 & $\mathrm{p}=0.01^{*}$ & 22 \\
Disturbed behaviour & 36 & & \\
\hline
\end{tabular}

NS $=$ Non significant. "Probability computed in a $t$ test comparing PTA in "low and medium" and "high" SB groups. Only 1 case in "low" SB group, so ANOVA impossible. 
jective burden scores over 5 years, and by the increasing reports of negative and distressing changes in the patient.

Frank violence against relatives had risen dramatically, and a number of relatives reported feeling afraid of the patient. Others reported constant anxiety as they tried to avoid provoking aggressive outbursts, and many felt that the patient could not be left alone. The types of change reported in the patient match closely those found by other workers. For example, Lezak ${ }^{10}$ described changes in social perceptiveness, self control, and childishness, which caused great distress to relatives, and Lewin, et al $^{16}$ found similar changes late after very severe injury. Thomsen's very late follow-up (10-15 years) found that even at that late stage "the psychosocial sequelae presented the most serious problems", ${ }^{17}$ but Weddell et $a l^{8}$ in a 2 year follow-up reported that most of the patients in their study were behaving in a "constructive and sensible fashion". Indeed, they reported specifically that there had been a reduction in irritability over the 2 years following injury, and an increase in affectionate behaviour. These results run contrary to those of others, and it is to be hoped that these positive findings may reflect the positive effects of rehabilitation, as all of Weddell's cases had been treated in a neurological rehabilitation centre.

Simple predictors of outcome in the patient and relative were sought, the most obvious one being the overall severity of injury: a predictor reported by almost all workers in this area. Certainly there were positive correlations between the level of change in the patient and the duration of post traumatic amnesia at 1 year, but at 5 years only one of the seven coefficients was significant. The extent to which patient outcome, as assessed by a relative, was related to overall severity of injury, diminished from the first to the fifth year of injury. Factors other than severity must, therefore, become increasingly important as time progresses. Such factors are very difficult to identify with any accuracy, although workers have speculated that factors such as the premorbid personality of the patient and stability of the home background $;^{814}$ and the personality of the relative ${ }^{21}$ may all be important. The environment in which the patient finds himself must obviously be important if only because the greater the degree of strain upon those around the patient, the less competently they will be likely to deal with him. Many of the relatives examined reported very high levels of strain at 5 years (certainly much more than at 1 year), and for them, the situation had deteriorated markedly, as had their ability to cope with the late outcome in the patient.

The more severe the initial injury, the more likely the relative is to report high levels of stress, with one linking mechanism being increasing incidence of negative behaviour and personality change in the patients with the greater severities of injury. Despite such significant relationships (table 7), there is still considerable "unexplained" variance suggesting that factors other than the injury and consequent changes in the patient may also be important in the prediction of burden. Such a factor may be the personality of the relative herself. Indeed, this has been examined within the first one year after injury when it emerged that the greater the "Neuroticism" score of the relative early after injury, the more likely that relative was to report high levels of stress. ${ }^{21}$

It is worth trying to specify routes by which changes in the patient as reported by a relative, increase over 5 years. There are a number of possible mechanisms at work, including attribution, sensitisation by the relative, and reactive changes in the patient. The process of attribution is one in which behaviour late after injury is interpreted as resulting from the injury, even though the causal link may be very tenuous. An example might be the high incidence of criminal convictions found over 5 years after injury, which would be very easy to attribute directly to the injury. Such an attribution would almost certainly be fallacious as in many cases criminal records existed even before the injury. Not only is attribution likely to be a problem for researchers in this area, but the very process of researching late outcome with its stress upon questioning about a variety of behavioural and other deficits may directly or indirectly lead to attribution. Relatives may have noticed behavioural features in the patient, and may not have considered them to be injury related, until asked specifically about them by a researcher.

A second mechanism for the late increase in behaviour disturbance may be sensitisation by a relative, who with increasing experience of interacting with the injured patient comes to identify problems that had been brushed aside or considered to be insignificant or denied earlier after injury. Examples here might be the communication and speech disturbances, and memory problems reported with greater frequency at 5 years than at one year. A related process is one in which the relative's threshold for tolerance of negative behaviour changes, so that behaviours acceptable and unremarked when infrequent, become unacceptable and very disturbing when frequent.

In the case of negative behaviours (aggression, etc), the reports of a late increase in problems may result not (or not solely) from an increased sensitisation or. attribution, or a changed threshold of tolerance in the relative, but from a genuine, reactive change in the patient's behaviour, as he attempts to deal with serious distressing changes in his life situation, and his 
future prospects for work, leisure, and social interaction.

Our research did not attempt to specify the precise mechanisms of late changes, and further research could with great profit look at the precise means by which behaviours change in frequency late after injury, and the appropriate measures which might be adopted to reduce the burden both for the patient, and his family.

LC, CS and AB were supported by Research Grant No. K/OPR/2/2/C610 from Scottish Home \& Health Department.

\section{References}

${ }^{1}$ Jennet B, Teasdale G. Management of head injuries, 1981. Philadelphia: FA Davies.

${ }^{2}$ Teasdale G, Mendelow D. Pathophysiology of head injuries. In: Brooks N, ed. Closed head injury: Psychological, Social and Family Consequences. Oxford: OUP, 1984;Ch 2:4-36.

${ }^{3}$ McKinlay WW, Brooks DN, Bond MR, Martinage DP, Marshall $M$. The short-term outcome of severe blunt head injury as reported by relatives of the injured persons. J Neurol Neurosurg Psychiatry 1981;44:527-33.

${ }^{4}$ Brooks DN, McKinlay W. Personality and behaviour change after severe blunt head injury: a relative's view. J Neurol Neurosurg Psychiatry 1983;46:336-44.

${ }^{5}$ Oddy M, Humphrey M, Uttley D. Stresses upon the relatives of head injured patients. $B r J$ Psychiatry 1978;133:507-13.

${ }^{6}$ Rosenbaum M, Najenson T. Changes in life pattern and symptoms of low mood as reported by wives of severely brain injured soldiers. $J$ Consult Clin Psychol 1976;44:881-8.

${ }^{7}$ Oddy $\mathbf{M}$, Humphrey $\mathbf{M}$. Social recovery during the year following severe head injury. $J$ Neurol Neurosurg Psychiatry 1980;43:789-802.
${ }^{8}$ Weddell $R$, Oddy M, Jenkins D. Social adjustment after rehabilitation: a two year follow-up of patients with severe head injury. Psychol Med 1980;10:257-63.

${ }^{9}$ Van Zomeren AH, van den Burg W. Residual complaints of patients two years after severe head injury. $J$ Neurol Neurosurg Psychiatry 1985;48:21-8.

${ }^{10}$ Lezak MD. Living with the characterologically altered brain injured patient. J Clin Psychol 1978;39:592-8.

${ }^{11}$ Fahy TJ, Irving MH, Millac P. Severe head injuries: a six year follow-up. Lancet 1967;2:476-79.

${ }^{12}$ Lundholm J, Jepsen BN, Thornval G. The late neurological, psychological, and social aspects of severe traumatic coma. Scand J Rehab Med 1975;7:97-100.

${ }^{13}$ Najenson T, Mendelson L, Schechter I, David C, Minz N, Groswasser Z. Rehabilitation after severe head injury. Scand J Rehab Med 1974;6:5-14.

${ }^{14}$ Gilchrist E, Wilkinson M. Some factors determining prognosis in young people with severe head injuries. Arch Neurol 1979;36:355-9.

${ }^{15}$ Brown JC. Late recovery from head injury: case report and review. Psychol Med 1975;5:239-48.

${ }^{16}$ Lewin W, Marshall TF de C, Roberts AH. Long term outcome after severe head injury. $B r$ Med $J$ 1979; 2:1533-7.

${ }^{17}$ Thomsen IV. Late outcome of very severe blunt head trauma: a 10-15 year second follow-up. $J$ Neurol Neurosurg Psychiatry 1984;47:260-8.

${ }^{18}$ Livingston MG, Brooks DN, Bond MR. Three months after severe head injury: psychiatric and social impact on relatives. J Neurol Neurosurg Psychiatry 1985,(a). 48;870-5.

${ }^{19}$ Livingston MG, Brooks DN, Bond MR. Patient outcome in the year following severe head injury, and relatives' psychiatric and social functioning. J Neurol Neurosurg Psychiatry 1985,(b). 48;876-81.

${ }^{20} \mathrm{Grad}$ J, Sainsbury P. Mental illness and the family. Lancet 1963;i:544-7.

${ }^{21}$ McKinlay WW, Brooks DN. Methodological problems in assessing psychosocial recovery following severe head injury. J Clin Neuropsychol 1984;6:87-99. 